\title{
Shakespeare y los leones: posibles encuentros entre inteligencia artificial y humanidades
}

Jackeline Bucio García

\section{Resumen}

En este artículo se comentan posibilidades de colaboración de grupos numerosos, con el apoyo de inteligencia artificial, en el área de las humanidades. Se hace énfasis en el impacto positivo que este tipo de colaboración puede lograr, a diferencia de la visión de confrontación que caracteriza el discurso actual sobre los peligros de la inteligencia artificial.

Palabras clave: colaboración masiva; aprendizaje, inteligencia artificial, supermentes, investigación

\section{SHAKESPEARE AND THE LIONS: POSSIBLE ENCOUNTERS BETWEEN ARTIFICIAL INTEL-} LIGENCE AND HUMANITIES

\begin{abstract}
This article is about the possibilities of collaboration among groups, with the support of artificial intelligence in Humanities. Emphasis is placed on the positive impact that this type of collaboration can achieve, unlike the confrontational vision that characterizes the current discourse on the dangers of artificial intelligence.
\end{abstract}

Keywords: crowdsourcing, artificial intelligence, superminds, people-powered research. 
"Shakespeare y los leones: posibles encuentros

Licenciada en Lengua y Literaturas Hispánicas (UNAM), maestra en Estudios de Asia y África (El Colegio de México) y doctora en Lingüística (UNAM).

Actualmente colabora como subdirectora de B@unam en la Coordinación de Universidad Abierta y Educación a Distancia de la unAm. Sus intereses de investigación se centran en formación docente en línea, innovación curricular, ciberpragmática, anotación digital, sistemas de tutoría inteligente, así como la edición de Wikipedia en contextos educativos.

Al pensar en inteligencia artificial (IA), uno de los imaginarios más comunes es el de la confrontación entre humanos y robots. Se habla de una inevitable guerra, de sometimiento, de "ellos o nosotros". Sobre estos peligros y preocupaciones que genera la integración de inteligencia artificial en nuestras vidas hay ya abundante discusión, y es muy importante que así sea. No obstante, autores como Dowek (2018) tienen otra perspectiva e invitan a salir de esa metáfora: "No hay más competencia entre el hombre y la máquina que entre el trompetista y la trompeta, el albañil y la pala, el escriba y el cálamo" (p. 248).

El temor a la sustitución, al relevo que un robot podría tomar del trabajo del ser humano, no nos permite bajar fácilmente las defensas, aun conociendo los beneficios que la inteligencia artificial está logrando en ámbitos como la medicina. Este miedo al reemplazo también permea el campo de las humanidades, pues la inteligencia artificial ya ha demostrado que puede crear, de manera autónoma, obras de arte o música, acciones que nos hacen considerar si a corto plazo el artista será sencillamente reemplazado. Alejándonos de esta perspectiva, la intención de este texto es explorar cómo la ı puede potenciar lo humano y el trabajo en colectivos para fomentar el logro de mejores resultados en proyectos relacionados con las humanidades. El escenario de la colaboración es una alternativa a la metáfora de la guerra, y es también la búsqueda de una productiva relación instrumental entre el ser humano y la IA, una herramienta ciertamente poderosa.

Podemos pensar que ya colaboramos con la tecnología de manera intensa, pero esto aún puede alcanzar un nuevo nivel si pensamos en lo que Malone denomina supermentes, es decir, la posibilidad de que "las personas y las computadoras se conecten de tal manera que actúen de forma más inteligente que cualquier persona, grupo o computadora haya hecho antes" (2018, p. 16). ¿Cómo puede suceder esto? Veamos algunos ejemplos. 


\section{Robots y humanos trabajando codo a codo}

Como ejemplo de robot y humanos trabajando juntos para lograr supermentes, Malone (2018) menciona a los bots de Wikipedia, diseñados para revertir ciertas ediciones consideradas como vandálicas. La inteligencia artificial detecta la anomalía y los bots contribuyen como aliados que auxilian a los editores humanos a mantener, de manera más sofisticada y rápida, la calidad de los artículos. Vista así, la inteligencia artificial, trabajando al servicio de un colectivo, potencia las habilidades de los miembros del grupo en lugar de sustituirlos, lo cual corresponde a uno de los "santos griales" de la IA (Weld, Mausam, Lin y Bragg, 2015, p. 89), propuesta también conocida como sistemas de iniciativa mixta.

Otro ejemplo de este tipo de colaboración es el proyecto EyeWire. Se trata de una plataforma de ciencia ciudadana que nació en 2012 y que permite la cooperación de voluntarios para mapear neuronas en un ambiente ludificado. Se han registrado más de 290000 personas de alrededor de 150 países y de acuerdo con la directora ejecutiva esto "es equivalente a 32 personas trabajando tiempo completo durante 7 años" (DeWeerdt, 2019).

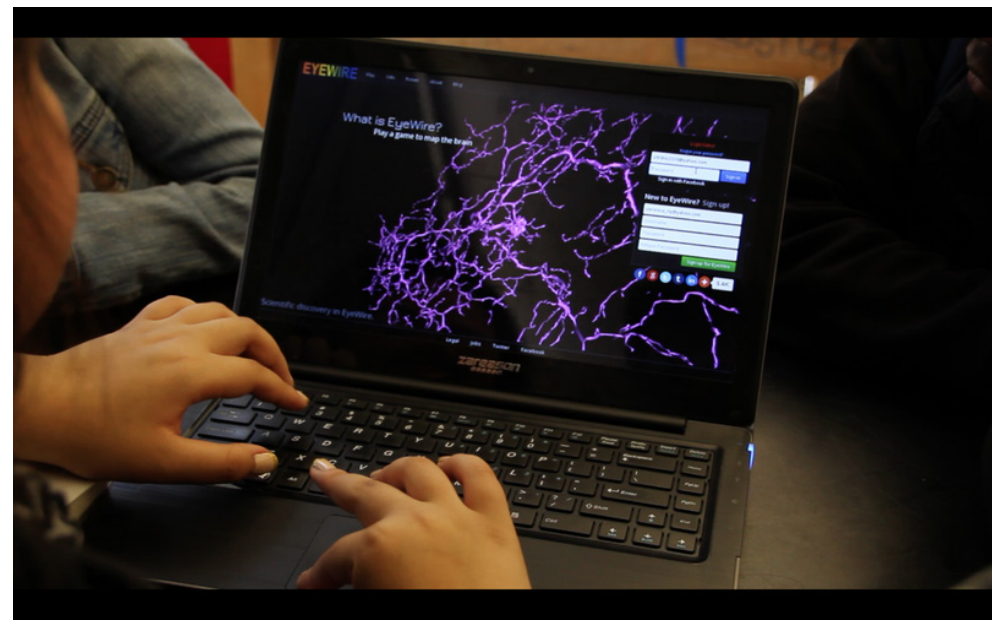

Figura 1. Plataforma de EyeWire

Nicholas Carr (2014) habla de la tecnología como un medio que nos permite ir más allá del límite que el cuerpo nos impone, y con ello nos acerca a una apreciación diferente del mundo: "El valor de una herramienta bien hecha y bien utilizada reside no sólo en lo que produce para nosotros, sino en lo que produce en nosotros (p. 247-248). También precisa precisa que la elección de una herramienta de trabajo debería ayudarnos a extender el campo de nuestra experiencia y percepción: "Lo que hace una herramienta superior a otra no tiene nada que ver con su novedad, lo que importa es cómo nos agranda o empequeñece, cómo moldea nuestra experiencia de la naturaleza, de la cultura y de los unos con los otros" (p. 262). 
Si asumimos las herramientas tecnológicas como un instrumento para enriquecer nuestra experiencia sensorial y cognitiva, no podríamos encontrar un mejor aliado que la ia para ayudarnos a conocer y mejorar el mundo que nos rodea. Los siguientes dos ejemplos muestran cómo este enriquecimiento de recursos puede aplicarse a casos concretos en las humanidades.

\section{El mundo de Shakespeare}

Zooniverse es el nombre de un proyecto que inició en 2009 bajo el concepto de people-powered research. Se trata de una plataforma que facilita la colaboración entre participantes de diferentes edades, países y conocimientos, permitiendo que cada uno aporte pequeñas cantidades de trabajo voluntario en diversos proyectos, bajo el mismo concepto que Wikipedia o EyeWire. De esta manera se avanza más y de forma más rápida. Al momento de escribir este texto, la página oficial anuncia 447339366 participaciones, en alrededor de 50 proyectos, hechos por 1937067 voluntarios registrados y contando. Otro término relacionado con dicha forma de trabajo es el crowdsourcing, que en este contexto se refiere a un llamado abierto a la colaboración en proyectos específicos, donde cada participante usa sus mejores habilidades con el fin de solucionar un problema determinado o resolver un reto. Al abrir una convocatoria de esta naturaleza se amplían las posibilidades de reunir perfiles variados que contribuyan de una forma que no sería posible en grupos cerrados y muy controlados.

Zooniverse alberga proyectos de todas las áreas del conocimiento, pero aquí nos centraremos en uno del área de humanidades llamado "El mundo de Shakespeare", donde la comunidad participa en la transcripción de manuscritos de este autor o de sus contemporáneos. El proyecto estuvo activo de diciembre de 2015 hasta septiembre de 2019 y actualmente se encuentra en período de revisión. Se trata de una iniciativa que se logra gracias a instituciones académicas como la Biblioteca Folger Shakespeare, en Washington, D.C., el proyecto Zooniverse.org de la Universidad de Oxford y el Diccionario Oxford de la Oxford University Press. Participaron alrededor de 50 mil usuarios que transcribieron aproximadamente 8000 páginas (Barber, 2018).

Entre los manuscritos por revisar se encontraban cartas, libros de recetas de cocina, textos de alquimia, boletines de noticias, etcétera. Este material permite identificar desde variantes lingüísticas del idioma inglés, hasta conocimientos sobre la vida cotidiana del período 1564-1616, cuyo contenido es casi imposible de analizar hasta no contar con una transcripción. Recordemos que para transcribir estos documentos se requieren especialistas y se trata de un proceso lento y minucioso que a una sola persona puede tomarle meses o años. A través de la plataforma que ofrece Zooniverse, este procedimiento se agiliza de la manera que se describirá a continuación. 
De entrada, todo el material se encuentra digitalizado y disponible en la plataforma. Los participantes realizan un tutorial de 10 minutos para aprender cómo deben indicar, desde una transcripción simple, hasta observaciones sobre palabras o expresiones no registradas aún por el diccionario, dudas o caracteres especiales. El sistema permite que cada voluntario colabore con una transcripción tan breve como una palabra o páginas completas: todo aporta al proyecto. En la figura 2 se puede apreciar el espacio que se abre sobre la imagen digitalizada de un manuscrito para ingresar una transcripción del texto, marcado con una línea verde.

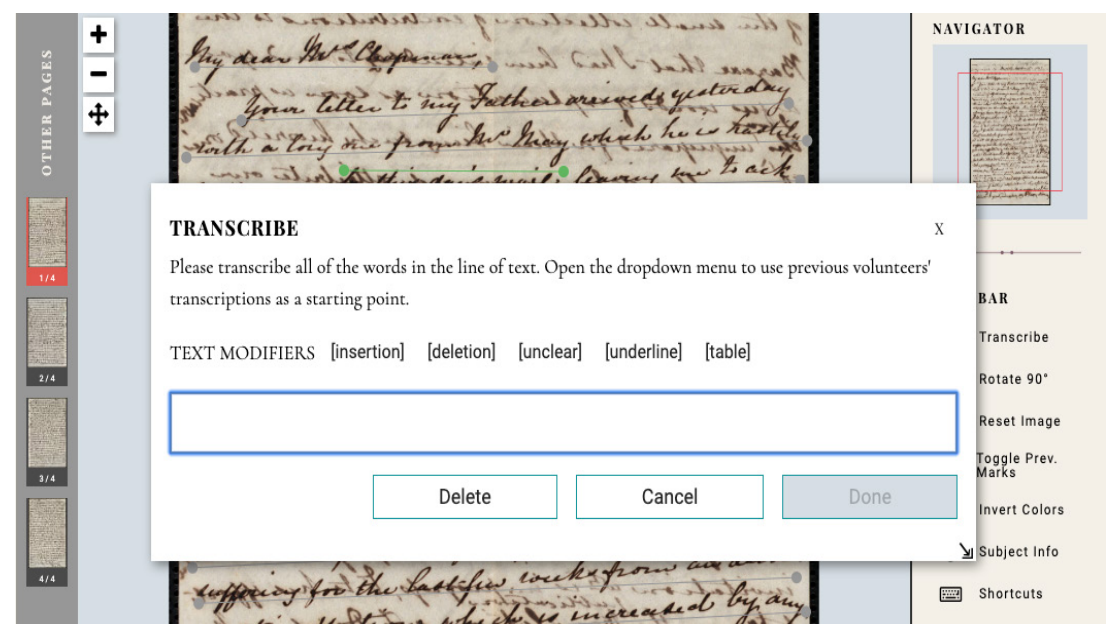

Figura 2. Plataforma Zoouniverse: ejemplos de transcripción.

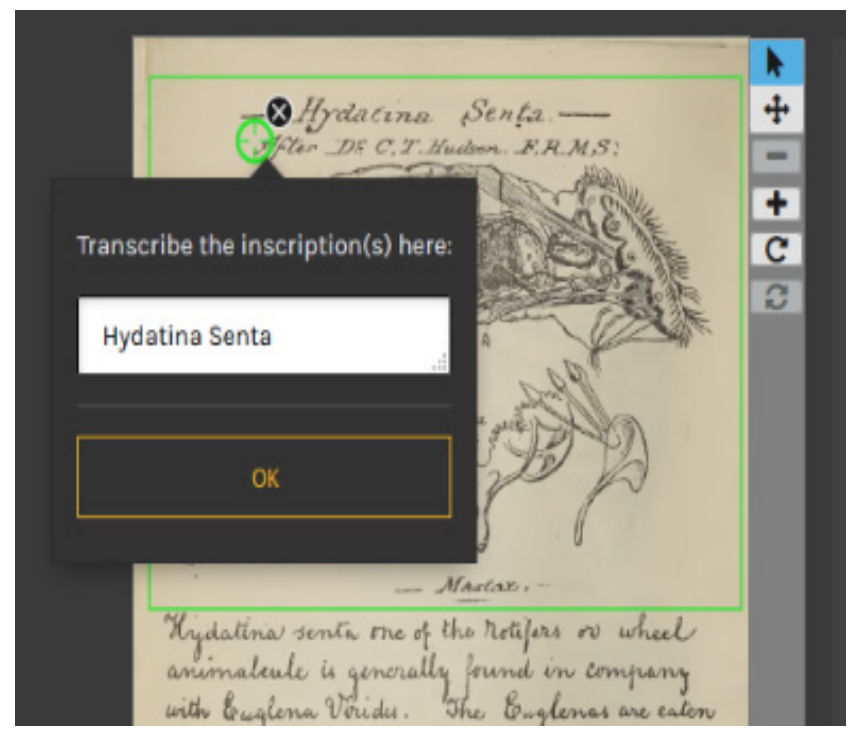

Por supuesto, no todas las participaciones son exactas y precisas, pero justamente aquí se encuentra la oportunidad para la inteligencia artificial: Ios algoritmos "aprenden" y podrán organizar, de manera cada vez más efectiva, la colaboración de toda la gente que decide participar en un proyecto de esta naturaleza, lo cual sería imposible si no fuera por los algoritmos que trabajan para facilitar la recuperación, análisis y validación de aportaciones. Aspectos como el reconocimiento de imagen a través de un entrenamiento de machine learning podrían mejorar la "lectura" que una máquina realiza de un manuscrito. 
"Shakespeare y los leones: posibles encuentros entre inteligencia artificial y humanidades "

Jackeline Bucio García

Vol. 21, Núm. 1, enero-febrero 2020

Revista Digital Universitaria

Los datos obtenidos quedan a disposición del público que así lo solicite y esta es una forma de retribución al trabajo voluntario y a la comunidad. Dunn y Hedges (2019), así como Deines, Gill, Lincoln y Clifford (2018) detallan los beneficios y retos que han observado de este tipo de colaboración. Estos últimos autores resaltan, en el primer punto de su análisis, un hecho obvio pero a la vez sorprendente: "Lección \#1: la gente quiere transcribir".

Entonces, las multitudes que leen a Shakespeare aportan la habilidad humana de lectura que las máquinas no poseen, mientras que las máquinas aportan la capacidad de clasificación y organización para sistematizar los esfuerzos del colectivo, sistema que podría verse beneficiado con el uso de IA. Tenemos aquí un ejemplo de supermente orientada hacia un proyecto del área de humanidades.

\section{Por favor, alimente a los leones}

El segundo ejemplo se trata de la colaboración entre Es Devlin, artista plástica, y Ross Goodwin, ingeniero de Google y creador de un algoritmo generador de poesía de deep learning para esta instalación. Please, Feed the Lions es el título original de esta obra que se presentó entre el 18 y 23 de septiembre de 2018, en el Festival de diseño de Londres. La idea era integrar un quinto león de color rojo vibrante que se uniera a los cuatro ya existentes en la plaza Trafalgar e invitar a los asistentes a alimentar a los leones..., pero con palabras. Aquí la metáfora de alimentar y nutrir a los leones nos regresa al aspecto de la colaboración para lograr mejores productos y de un mayor impacto. Podemos preguntarnos en este punto, ¿con este "alimento" aportado por la gente, qué tipo rugido produciría el león? Al concebir esta obra, Devlin pensaba darle al león "una voz poética colectiva y diversa a partir del 'alimento' proporcionado por las multitudes" (Google Arts \& Culture, 2018).

Dado que la elección de las palabras que una persona usaría para alimentar a los leones era un factor desconocido, el algoritmo debía estar diseñado para optimizar la estética del poema tanto en lo semántico como en la métrica.

${ }^{1}$ This shelter in the wood

The sun and sky

The shadows of the day do shake the skies And feel the precious sounds of death are

A despot still upon the shores of $\sin$

And the stars of the night-wind seem to stand

El poema completo se puede leer aquí. Cada palabra o frase que llegaba a la boca del león se convertía en el inicio de un poema de dos líneas que se proyectaba en la columna cercana. Estos son algunos de los versos que el león rugió:

Este refugio en el bosque

El sol y el cielo

Las sombras del día sacuden los cielos

Y siente los preciosos sonidos de la muerte ser

Un déspota aún sobre las costas del pecado

Y las estrellas del viento nocturno parecen estar de pie ${ }^{11}$ 
Figura 3. Please. Feed the Lions, Trafalgar Square, Londres 2018 (Mac Fadden Islington, 2018).

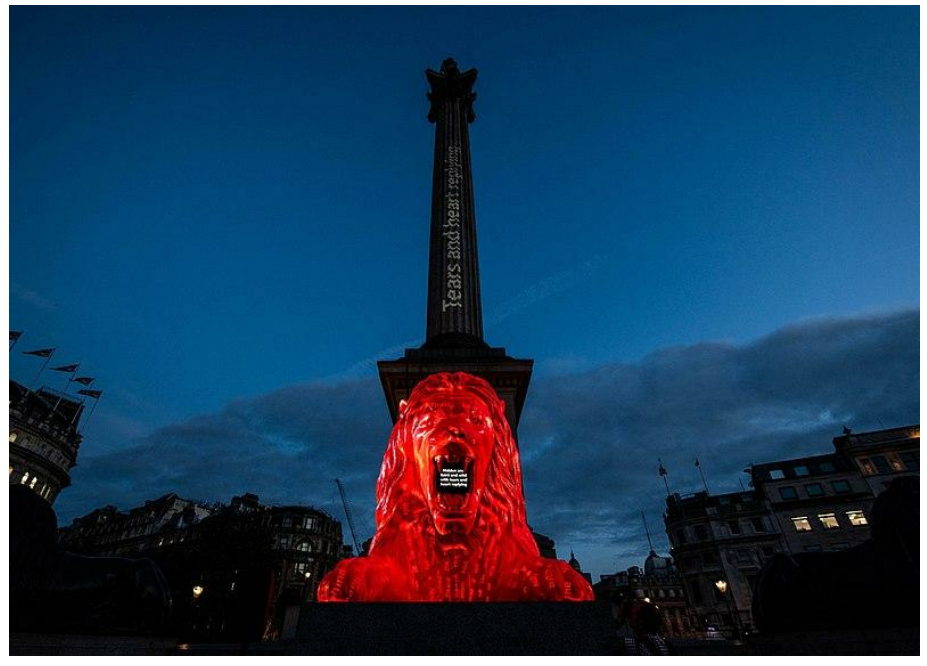

El poema se genera a partir de un modelo de red neuronal que ha aprendido a escribir a través de la lectura de 25 millones de palabras de poesía del siglo xIx. Funciona prediciendo el carácter que debe continuar (sea letra, espacio o puntuación), a partir de cada una de las palabras proporcionadas por la audiencia. El algoritmo no sólo une frases que conoce con las que recibe, sino que puede escribir por sí mismo a partir de un input determinado: "Esto permite a la obra responder en tiempo real, es un poema siempre en desarrollo" (Google Arts \& Culture, 2018).

En este caso, la inteligencia artificial permite que las multitudes creen un poema que además de extenso es verdaderamente misterioso en su sentido. Si cada persona en este festival escribiera su propio poema sería una creación valiosa sin duda, pero el acto de crearlo de manera colectiva y con la posibilidad de mejorar y potenciar la estética del lenguaje gracias al algoritmo es otro ejemplo de las supermentes a las que se refiere Malone (2018).

\section{Lavisión positiva de la IA, las multitudesy lashumanidades}

Si regresamos a la definición de Carr (2014) acerca de la productividad de una herramienta que no sólo produce algo para nosotros sino que produce algo (positivo) en nosotros y nos permite extender nuestras posibilidades de experiencia del mundo, los dos ejemplos toman sentido. Lejos de la ia que nos sustituya o que nos haga cada vez más dependientes e inútiles al recordar datos, en estos dos ejemplos vemos una perspectiva optimista de las posibilidades de colaboración: si la metáfora de competencia cambia por una de "alimentación", de muchos que podemos aportar, cada uno, un poco para lograr más entre todos, entonces, tal vez podamos lograr una balanza más equilibrada, positiva y proactiva: las humanidades tienen abundante material para nutrir estas supermentes y aprender en ellas. 


\section{Referencias}

* Barber, S. T. (2018). The ZOONIVERSE is Expanding: Crowdsourced Solutions to the Hidden Collections Problem and the Rise of the Revolutionary Cataloging Interface. Journal of Library Metadata, 18(2), 85-11. Dol: https://doi.org/10.1080/193 86389.2018.1489449.

* Carr, N. (2014). Atrapados. Cómo las máquinas se apoderan de nuestras vidas. México, CDMX: Taurus.

- Deines, N., Gill, M., Lincoln, M. y Clifford, M. (2018). Six Lessons Learned from Our First Crowdsourcing Project in the Digital Humanities. Getty Iris blog. Recuperado de: https://blogs.getty.edu/iris/six-lessons-learned-from-our-firstcrowdsourcing-project-in-the-digital-humanities.

* DeWeerdt, S. (24 de julio de 2019). How to map the brain. Nature Outlook: The brain, 571, S6-S8 doi: 10.1038/d41586-019-02208-0

- Dowek, G. (2018). Entrevista en Intelligence artificielle. Enquête sur ce technologies qui changent nos vies. Flammarion, Champs actuel.

Dunn, S. y Hedges, M. (2017). Academic Crowdsourcing in the Humanities. Crowds, communities, and Co-production. Cambridge, Massachusetts: Chandos PublishingElsevier.

Google Arts \& Culture (2018). The Technology and people behind Please Feed the Lions. Meet the team working behind-the-scenes on the fifth lion. Recuperado de: https://artsandculture.google.com/exhibit/uQJy-TQgHNPkJw.

* Mac Fadden Islington, Dearbhla (2018). "Please Feed The Lions", Es Devlin in Trafalgar Square, London 2018. Recuperado de: https://commons.wikimedia.org/ wiki/File:LDFLionsEdit 06.jpg .

* Malone, T. (2018). Superminds: The Surprising Power of People and Computers Thinking Together. Nueva York, Little, Brown and Company.

* Weld, D., Mausam, Lin, C. y Bragg, J. (2015). Artificial Intelligence and Collective Intelligence, p. 89-100 En Malone y Bernstein (eds). Handbook of collective intelligence. Boston, Massachusetts: mit Press.

\section{Cómo citar este artículo}

* Bucio García, Jackeline (2020). Shakespeare y los leones: posibles encuentros entre inteligencia artificial y humanidades. Revista Digital Universitaria (RDU). Vol. 21, núm. 1 enero-febrero. Dol: http://doi.org/10.22201/codeic.16076079e.2020.v21n1.a4.

Recepción: 06/11/2019. Aprobación: 22/11/2019 